\title{
Stress Analysis of an undulated plate by the Combined First Order Perturbation and Alternating Method
}

\author{
by Yoichi Sumi*, member and Ahmad Rahbar-Ranji**, student member
}

\begin{abstract}
Summary
A new solution method is proposed for the analysis of an undulated plate of finite thickness subjected to in-plane loading conditions. The method treats the boundary conditions on the undulated surfaces by using a first order perturbation method, where the deviation of the undulating surface from a reference plane surface is treated as the small perturbation parameter of the problem. In order to satisfy the boundary conditions on the top and bottom surfaces, solutions of a semi-infinite body are alternately applied, where the solution procedure utilizes relatively simple analytical expressions without tedious numerical calculations such as arising in the finite element analyses. It should be noted that an arbitrarily undulated surface of a plate may be approximated by the superpostion of two $)^{-}$ dimensional sinusoidal waves having arbitrary wave lengths, directions and phases. This means that only certain two dimensional solutions are necessary to construct a fully three-dimensional solution. The solution accuracy of the proposed method is examined by comparing the present results with finite - element solutions, and illustrative examples are also given for the stress analysis of a plate with slightly wavy surfaces in three-dimension.
\end{abstract}

\section{Introduction}

Structural failures often start from irregular surfaces caused by imperfection due to welding process or corrosion during their service lives. Although numerical methods such as the finite-element method are used for stress analyses, these methods are usually very tedious and time-consuming to calculate the stress distribution near the irregular surface because of the considerably different length scales involved in the problem, i. e.; the so-called multi-scale problem. In order to overcome this difficulty, a semi-analytical approach based on the combined first order perturbation and alternating method is proposed in this paper, where attention is focused on the stress analysis of plates of finte thickness with undulaing surfaces.

Stress concentration at slightly undulating surfaces had been investigated by (Gao ${ }^{11}$ using the first order perturabation method, in which undulating surfaces are perturbed from a reference state which is perfectly flat. He applied the method to a half plane problem in two -dimension and half space problem in three-dimension. Later, a second order perturbation method is applied to

* Faculty of Engineering, Yokohama National University.

** Graduate School of Engineering, Yokohama National University.

Received 10 th Jan. 20()1

Read at the Spring meeting 17, 18 th May 2001 two-dimensional problems by Sumi ${ }^{2)}$ for the accurate determination of stress gradient and stress distribution in stress concentrated regions. In the present paper a new combined first order perturbation and alternating method is proposed for the analysis of stress concentration in a plate with slightly undulating surfaces, where the perturbation method is applied to satisfy the boundary conditions due to undulating surfaces, while the Schwartz-Neumann alternating method (Kantorovich and $\mathrm{Krilov}^{3)}$ ) is used to take into account of the effect of the back surface, respectively.

It is interesting to note that the stress caused by an arbitrarily undulating surfaces may be approximated by the linear superposition of those arising from regular sinusoidal surfaces having arbitray wave length and wave direction subjceted to in-plane tension-compression and shear because of the linearity of the response with regard to the perturbed surface geometry. This means that we only need the first-order solution of a two-dimensional sinusoidal surfaces subjected to inplane tension and anti plane shear as the fundamental solutions in order to solve a problem having arbitrary three-dimensional surfaces.

This paper is organized in the following manner. In Section 2 the plane problem having undulating surfaces is defined. A general formulation of the combined first order perturbation and alternating method will be presented in Section 3. In Section 4 the solution of a fully three-dimensional problem is expressed by the integration of two-dimensional solutions, which is obtained in a closed form. Finally in Section 5 some 
illustrative examples are given so that discussions will be made for the validity and applicability of the present method.

\section{Statement of Problem}

\section{1 Plate with undulating surfaces}

We shall consider a plate with undulating surfaces subjected to combined biaxial tension and shear loading. The Cartesian coordinate system $O-x_{1} x_{2} x_{3}$ is defined in such a manner that the middle surface of the plate coincides with the $x_{1} x_{2}$-plane (see Fig. 1). The undulating top and bottom surfaces of the plate are expressed by a pair of functions, $h^{+}\left(x_{1}, x_{2}\right)$ and $h^{-}\left(x_{1}\right.$, $\left.x_{2}\right)$, respectively, where the averaged levels of the top and bottom surfaces, $\bar{h}^{ \pm}$satisfy the following conditions:

$$
\overline{h^{ \pm}} \equiv \frac{\iint_{S_{0}} h^{ \pm}\left(x_{1}, x_{2}\right) d S}{\iint_{S_{0}} d S}= \pm \frac{t}{2},
$$

where $S_{0}$ indicates the middle surface of the plate, which locates on the plane $x_{3}=0$. Eqn. (1) results in the averaged thickness of $t$ of the plate. At this moment we simply assume that the functions $h^{ \pm}\left(x_{1}, x_{2}\right)$ is continuous and at least differentiable once with respect to $x_{1}$ and $x_{2}$. We shall also introduce a pair of the following functions corresponding to $h^{ \pm}\left(x_{1}, x_{2}\right)$;

$$
\zeta_{0}^{ \pm}\left(x_{1}, x_{2}\right) \equiv h^{ \pm}\left(x_{1}, x_{2}\right) \mp \frac{t}{2}
$$

whose order is assumed to be small compared with the averaged thickness, $t$, i. e. $\left|\zeta_{0}^{ \pm} / t\right| \ll 1$. Then we can naturally represent an arbitrarily undulating surface in terms of a kind of Fourier integral:

$$
\begin{aligned}
& \zeta_{0}^{ \pm}\left(x_{1}, x_{2}\right)=\int_{0}^{\infty} \int_{0}^{\pi} A^{ \pm}(\lambda, \psi) \cos \\
& {\left[\frac{2 \pi}{\lambda}\left(x_{1} \cos \psi+x_{2} \sin \psi\right)+\varphi(\lambda, \psi)\right] d \psi d \lambda}
\end{aligned}
$$

where $A^{ \pm}(\lambda, \psi)$ is the amplitude of a sinusoidal wave with its wave length, $\lambda$, and its directional angle $\psi$ to the $x_{1}$-axis, and its phase angle, $\varphi(\lambda, \psi)$, (see Fig. 2).

\section{2 Statement of Problem}

The plate is subjected to in-plane loads so that the problem can be expressed by the following governing equation and boundary conditions;

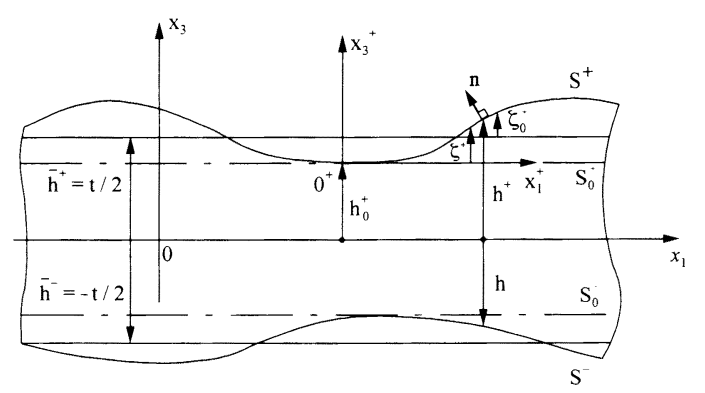

Fig. 1 Undulated plate with various coordiante systems for use in the analysis.

$$
\begin{aligned}
& \sigma_{i j, j}=0 \text { in } \mathrm{V} \\
& \sigma_{i j} n_{j}=0 \text { on } S^{ \pm} \\
& \frac{\iint_{S_{1}{ }^{ \pm}} \sigma_{i j} n_{j} d S}{\iint_{S_{1}} d S}=\left\{\begin{array}{ll} 
\pm \sigma_{1} & \mathrm{i}=1 \\
\pm \tau & \mathrm{i}=2
\end{array} \text { on } S_{1}^{+}\right. \\
& \frac{\iint_{S_{2} \pm} \sigma_{i j} n_{j} d S}{\iint_{S_{2}} d S}=\left\{\begin{array}{ll} 
\pm \tau & \mathrm{i}=1 \\
\sigma_{2} & \mathrm{i}=2
\end{array} \text { on } S_{2}^{+}\right.
\end{aligned}
$$

where $\mathrm{V}$ indicates the domain of plate, $S_{1}^{ \pm}$and $S_{2}^{ \pm}$ represent the surfaces of the four edges of the plate with $x_{1}=$ const., and $x_{2}=$ const., respectively, and the surfaces, $S^{ \pm}$, indicate the top and bottom surfaces of the plate (see Fig. 3). The unit outward normals on the surfaces are denoted by $n_{i}(i=1,2,3)$.

Having defined the boundary conditions, the problem is now to find out the stress at a point $O^{+}\left(x_{1}^{*}, x_{2}^{*}, h_{0}^{+}=\right.$ $\left.h\left(x_{1}^{*}, x_{2}^{*}\right)\right)$ as illustrated in Fig. 1, where high stress

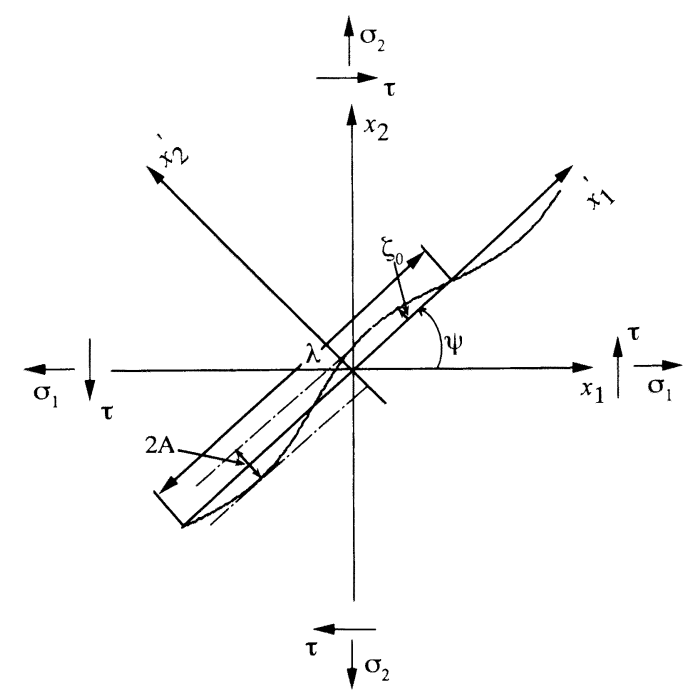

Fig. 2 An arbitrary regular wave component with wave-direction $\phi$.

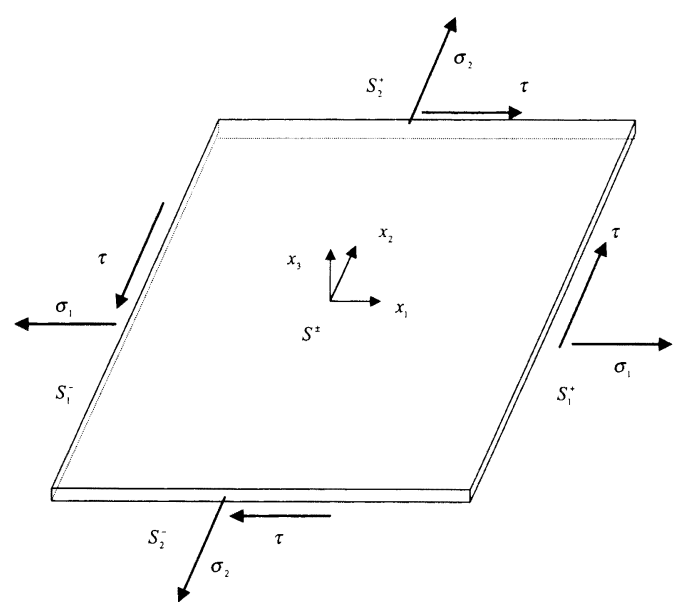

Fig. 3 Loading condition of an undulated plate. 
concentration could be expected.

\section{Method of Solution}

3. 1 First order perturbation solution of a finite thickness plate with undulated surfaces

In order to solve the problem defined in the previous section, we shall begin with the first order perturbation analysis of a finite thickness plate with undulating top and bottom surfaces. Stresses in the vicinity of the point of interest, $\mathrm{C}^{+}$, can be expressed by the following form :

$$
\sigma_{i j}=\sigma_{i, j}^{(0)}+\sigma_{i, j}^{+(1)}+\sigma_{i, j}^{-(1)}+O\left(\zeta^{2}\right)
$$

where $\sigma_{i, j}^{(0)}$ and $\sigma_{i, j}^{+(1)}$ are of the zero-th and first order with respect to $\zeta^{ \pm}\left(x_{1}, x_{2}\right)$. The first order stresses in Eqn. $(8)$ are linear with respect to the small perturbation parameter, $\zeta^{ \pm}\left(x_{1}, x_{2}\right)$, because the perturabation is of the first order in the present formulation. First, we shall try to satisfy the boundary conditions on the undulated surfaces,

$$
\sigma_{i j} n_{j}=0 \quad \text { on } S^{+}
$$

where stresses $\sigma_{i j}$ and the external unit normal vector $n_{i}$ on $S^{ \pm}$are expanded in terms of $\zeta^{ \pm}$up to the first order on the flat reference surfaces, $S_{0}^{+}$:

$$
\begin{aligned}
& \sigma_{i j}=\sigma_{i, j}^{(0)}+\sigma_{i, 3,3}^{(0)} \zeta^{ \pm}+\sigma_{i, j}^{+(1)}+\sigma_{i,}^{-(1)}+O\left(\zeta^{2}\right) \\
& n_{1}=\mp \zeta, \overline{1}, n_{2}=\mp \zeta, \zeta_{, 2}, n_{3}= \pm 1
\end{aligned}
$$

Substitution of Eqns. (10) and (11) into Eqn. ( 9 ) leads to the ordered boundary conditions for $\sigma_{i, j}^{(0)}$ and $\sigma_{i j}^{ \pm(1)}$ respectively, as :

$$
\begin{aligned}
& \sigma_{i 3}^{(0)}=0 \text { on } S_{0}^{+} \\
& \sigma_{i 3}^{+(1)}+\sigma_{i 3}^{-(1)}=\sigma_{i 1}^{(0)} \zeta_{.1}^{ \pm}+\sigma_{i 2}^{(0)} \zeta_{, 2}^{ \pm}-\sigma_{i 3,3}^{(0)} \zeta^{ \pm}
\end{aligned} \text {on } S_{0}^{ \pm}
$$

It should be noted that the boundary conditions given by (12) and (13) are defined on the flat reference surfaces, $S_{0}^{+}$, where $S_{0}^{+}$, passes through the point of interest, $O^{+}$.

Since the loading conditions are given by Eqns. (6) and $(7)$, we shall take a simple biaxial stress as the zero-th order solution, $\sigma_{i j}^{(0)}$ :

$$
\sigma_{i j}^{(0)}= \begin{cases}\sigma_{1} & i=j=1 \\ \sigma_{2} & i=j=2 \\ \tau & i=1, j=2 ; i=2, j=1 \\ 0 & \text { otherwise }\end{cases}
$$

which obviously satisfies all the boundary conditions for a flat plate, i. e. $\zeta^{ \pm}=0$. Furthermore, due to the linearity of the first order solution, we shall assume that the first order terms, $\sigma_{i j}^{ \pm(1)}$, are chosen so as to satisfy the following conditions ;

$$
\sigma_{i 3}^{ \pm(1)}=0 \text { on } S_{0}^{\mp} \text {. }
$$

Eqn. (13) then follows;

$$
\sigma_{i 3}^{ \pm(1)}=\sigma_{i 1}^{(0)} \zeta_{.1}^{ \pm}+\sigma_{i 2}^{(0)} \zeta_{.2}^{ \pm} \quad \text { on } S_{0}^{ \pm},
$$

which means that $\sigma_{i j}^{+(1)}$ is calculated in such a way that it satisfies the undulated surface conditions on the top or bottom surface, independently, assuming the opposite surface to be flat.

Since the same approach may be applied to the points of interest on the undulated bottom surface, we shall not duplicate the discussion here. We shall next consider the alternating solution scheme for the construction of the first order solution for a finite thickness plate.

\section{2 An alternating solution scheme for a finite thickness plate}

In this subsection we shall introduce an alternating solution scheme so as to satisfy the boundary conditions of the top and bottom surfaces. We shall begin with the first order solution, $\sigma_{i j}^{+(1)}$, of the following problem;

$$
\begin{aligned}
& \sigma_{i 3}^{+(1)}=\sigma_{i 1}^{(0)} \zeta_{, 1}^{+}+\sigma_{i 2}^{(0)} \zeta_{, 2}^{+} \quad \text { on } S_{0}^{+}, \\
& \sigma_{i 3}^{+(1)}=0 \text { on } S_{0}^{-},
\end{aligned}
$$

which corresponds to a plate having an undulated top surface with a flat bottom. Having introduced an alternating solution scheme, the first order solution for the finite thickness plate can be expressed by

$$
\sigma_{i j}^{+(1)}=\sigma_{i j}^{l(1)}\left(\zeta^{+}\right)+\sum_{k=0}^{\infty} \sigma_{i j}^{+[k, k+1]},
$$

where the stress $\sigma_{i j}^{+|k, k+1|}$ should be determined in such a way that the boundary conditions, Eqns. (17) and (18), are successively satisfied by solving the following boundary value problems :

$$
\begin{aligned}
& \sigma_{i 3}^{l(1)}=\sigma_{i 1}^{(0)} \zeta_{, 1}^{+}+\sigma_{i 2}^{(2)} \zeta_{, 2}^{+} \text {on } S_{0}^{+}, \\
& \sigma_{i 3}^{+[0,1]}=-\sigma_{i 3}^{l 1)} \text { on } S_{0}^{-}, \\
& \sigma_{i 3}^{+\{k, k+1]}=-\sigma_{i 3}^{+\{k-1, k]} \text { on } \begin{cases}S_{0}^{+} & \text {for } k=1,3,5, \cdots \\
S_{0}^{-} & \text {for } k=2,4,6, \cdots\end{cases}
\end{aligned}
$$

where $\sigma_{i j}^{l(1)}$ is obatined as the first order solution for the lower half-space with its surface, $S^{+}$, which satisfies the boundary conditions (17) on the top surface. Then the stress, $\sigma_{i 3}^{+\{0,1\}}$, is determined for the upper half-space with its surface, $S^{-}$, so that the boundary conditons (18) on the bottom surface is satified. In the alternating method, this process continues until the convergence of the solution is attained. In order to calculate the stresses, $\sigma_{i j}^{l(1)}$ and $\sigma_{i j}^{+\mid k, k+1]},(k=0,1,2, \cdots)$ we can simply integrate the applied forces on the surface by using the Boussinesq's fundamental solution of a point force acting on the surface of a semi-infinite half-space.

Similarly the first order solution, $\sigma_{i j}^{-(1)}$ for the undulated bottom surface is calculated by the alternating method as,

$$
\sigma_{i j}^{-(1)}=\sigma_{i j}^{u(1)}\left(\zeta^{-}\right)+\sum_{k=0}^{\infty} \sigma_{i j}^{-[k, k+1]},
$$

where

$$
\begin{aligned}
& \sigma_{i 3}^{u(1)}=\sigma_{i 1}^{(0)} \zeta_{, 1}^{-}+\sigma_{i 2}^{(0)} \zeta_{, 2}^{-} \text {on } S_{0}^{-}, \\
& \sigma_{i 3}^{-[0,1]}=-\sigma_{i 3}^{u(1)} \text { on } S_{0}^{+}, \\
& \sigma_{i 3}^{-[k, k+1]}=-\sigma_{i 3}^{-[k-1, k]} \text { on }\left\{\begin{array}{l}
S_{0}^{-} \text {for } k=1,3,5, \cdots \\
S_{0}^{+} \text {for } k=2,4,6, \cdots
\end{array}\right.
\end{aligned}
$$

The stress, $\sigma_{i j}^{u(1)}$, is the first order solution for the upper half-space with its surface, $S_{0}^{-}$, which satisfies the boundary conditions (16) on the bottom surface. Having obtained the all necessary first order solutions, the final form of the combined first order perturbation and alternating solution is given by Eqn. ( 8 ).

\section{Three-dimensional Solution Composed by Fundamental Two-dimensional Solutions}

\section{1 Two-dimensional solutions for a sinusoidal surface}

Considering the first order solution given by Eqn. ( 8 ) 
with its first order terms determined by the boundary conditions (16), we can easily see the linear characteristics of the solution with respect to $\zeta^{ \pm}$. Also, if we remind of the integral representation, Eqn. ( 3 ), of $\zeta_{0}^{ \pm}$, a general three-dimensional solution of the first order may be obtained by the integration of the two-dimensional solution for a plate with an arbitrary regular sinusoidal surface. In this subsection we shall obtain the solution of this fundamental two-dimensional problem, in which only one surface is assumed to be undulated in a simple sinusoidal shape.

Let us consider the problem of a regular sinusoidal surface as illustrated in Fig. 2, where the loading condition is given by Eqns. ( 6 ) and ( 7 ). By using the the coordinate system $O-x_{1}^{\prime} x_{2}^{\prime} x_{3}$, the averaged loading condition may be rewritten as

$$
\sigma_{i j}^{(0)}= \begin{cases}\sigma_{1}^{\prime} & i=j=1 \\ \sigma_{2}^{\prime} & i=j=2 \\ \tau^{\prime} & i=1, j=2 ; i=2, j=1 \\ 0 & \text { otherwise }\end{cases}
$$

where $\sigma_{1}^{\prime}, \sigma_{2}^{\prime}$ and $\tau^{\prime}$ can easily be obtained by the coordinate transformation of angle $\psi$. We shall begin with solving the first order boundary value problem given by the first equation of Eqn. (20) for a semi-infinite lower half-plane,

$$
\sigma_{i 3}^{l(1)}=\left\{\begin{array}{ll}
\sigma_{1}^{\prime} \zeta_{, 1}^{+1} & i^{\prime}=1 \\
\tau^{\prime} \zeta_{.1}^{+} & i^{\prime}=2 \\
0 & i^{\prime}=3
\end{array} \text { on } S_{0}^{+} .\right.
$$

If we use the in-plane and anti-plane solutions of point forces acting on $S_{0}^{+}$in the $x_{1-}^{-}$and $x_{2}^{\prime}$-directions, respectively, their convolution integrals with the right hand side of Eqn. (24) lead to stresses, $\sigma_{i j}^{l(1)}$. The inplane components of the stresses can be given by

$$
\begin{aligned}
& \sigma_{11}^{+l(1)}=\frac{4 \pi A}{\lambda} \sigma_{1}^{\prime}\left(1+\frac{\pi x_{3}^{+}}{\lambda}\right) \exp \left(\frac{2 \pi x_{3}^{+}}{\lambda}\right) \cos \\
& \left(\frac{2 \pi x_{1}^{\prime}}{\lambda}+\varphi\right), \\
& \sigma_{22}^{+l(1)}=\nu \frac{4 \pi A}{\lambda} \sigma_{1}^{\prime} \exp \left(\frac{2 \pi x_{3}^{+}}{\lambda}\right) \cos \left(\frac{2 \pi x_{1}^{\prime}}{\lambda}+\varphi\right), \\
& \sigma_{33}^{+l(1)}=-\frac{4 \pi A}{\lambda} \sigma_{1}^{\prime} \frac{\pi x_{3}^{+}}{\lambda} \exp \left(\frac{2 \pi x_{3}^{+}}{\lambda}\right) \cos \left(\frac{2 \pi x_{1}^{\prime}}{\lambda}+\varphi\right), \\
& \sigma_{13}^{+l(1)}=\frac{2 \pi A}{\lambda} \sigma_{1}^{\prime}\left(1+\frac{2 \pi x_{3}^{+}}{\lambda}\right) \exp \left(\frac{2 \pi x_{3}^{+}}{\lambda}\right) \\
& \sin \left(\frac{2 \pi x_{1}^{\prime}}{\lambda}+\varphi\right) .
\end{aligned}
$$

where $x_{3}^{+}$is the distance from the top reference surface, $S_{0}^{+}$, in the thickness direction, and defined by

$$
x_{3}^{+} \equiv x_{3}-h_{0}^{+}
$$

The anti-plane shear components are also calculated as

$$
\begin{aligned}
& \sigma_{12}^{+l(1)}=\frac{2 \pi A}{\lambda} \tau^{\prime} \exp \left(\frac{2 \pi x_{3}^{+}}{\lambda}\right) \cos \left(\frac{2 \pi x_{1}^{\prime}}{\lambda}+\varphi\right), \\
& \sigma_{23}^{+l(1)}=\frac{2 \pi A}{A} \tau^{\prime} \exp \left(\frac{2 \pi x_{3}^{+}}{\lambda}\right) \sin \left(\frac{2 \pi x_{1}^{\prime}}{\lambda}+\varphi\right) .
\end{aligned}
$$

According to the general procedure described in the previous section, the boundary conditions corresponding to the finite thickness plate will be satisfied by the alternating method described in the second and third equations of Eqn. (20) ;

$$
\sigma_{i 3}^{+[0,1]}=-\sigma_{i 3}^{l(1)} \text { on } S_{0}^{-} \text {. }
$$

$$
\sigma_{i 3}^{+|k, k+1|}=-\sigma_{i 3}^{+|k-1, k|} \text { on }\left\{\begin{array}{l}
S_{0}^{+} \text {for } k=1,3,5, \cdots \\
S_{0} \text { for } k=2,4,6, \cdots
\end{array}\right.
$$

The above alternating solutions can be obtained in the closed forms similar to Eqns. (25) and (27), where we additionally use the solution of a point force acting on $S_{0}^{ \pm}$in the $x_{3}$ direction. Substitution of Eqns. (25), (27), and the alternating solutions of Eqn. (28) into Eqn. (19) leads to the first order solution, $\sigma_{i, j}^{+(1)}$, which converges to a closed-form solution because of the exponential nature in the thickness direction.

The dominant in plane components of the final form of the first order solution of the fundamental two. dimensional solution are expressed by

$$
\begin{aligned}
& \sigma_{11}^{+(1)}=\frac{4 \pi A}{\lambda} \frac{\cos \left[(2 \pi / \lambda)\left(x_{1} \cos \psi+x_{2} \sin \psi^{\prime}\right)+\varphi\right]}{\sinh \left(2 \pi t_{0} / \lambda\right)} \\
& \left\{\left[\sigma_{1}^{\prime}\left(\cos ^{2} \psi+\nu \sin ^{2} \psi\right)+\frac{1}{2} \tau^{\prime} \sin 2 \psi^{\prime}\right]\right. \\
& \cosh \frac{2 \pi x_{3}^{-}}{\lambda}+\sigma_{1}^{\prime} \cos ^{2} \psi\left[\begin{array}{c}
\pi x_{3} \\
\lambda
\end{array} \sinh \left(\begin{array}{c}
2 \pi x_{3}^{-} \\
\lambda
\end{array}\right)\right. \\
& -\frac{\pi t_{0}}{2 \lambda}\left\{\exp \left(\begin{array}{c}
-2 \pi x_{3} \\
\lambda
\end{array}\right)\right. \\
& \left.\left.\left.+\frac{\exp \left(2 \pi x_{3}^{+} / \lambda\right)+\cosh \left(2 \pi t_{0} / \lambda\right) \exp \left(2 \pi x_{3} / \lambda\right)}{\sinh \left(2 \pi t_{0} / \lambda\right)}\right\}\right]\right\}, \\
& \begin{array}{cc}
\sigma_{22}^{+(1)}=\frac{4 \pi A}{\lambda} \cos \left[(2 \pi / \lambda)\left(x_{1} \cos \psi^{\prime}+x_{2} \sin \psi^{\prime}\right)+\varphi\right] \\
\sinh \left(2 \pi t_{0} / \lambda\right)
\end{array} \\
& \left\{\left[\sigma_{1}^{\prime}\left(\sin ^{2} \psi^{\prime}+\nu \cos ^{2} \psi^{\prime}\right)+\frac{1}{2} \tau^{\prime} \sin 2 \psi^{\prime}\right]\right. \\
& \cosh \frac{2 \pi x_{3}^{-}}{\lambda}+\sigma_{1}^{\prime} \sin ^{2} \psi^{\prime}\left[\begin{array}{c}
\pi x_{3} \\
\lambda
\end{array} \sinh \left(\begin{array}{c}
2 \pi x_{3} \\
\lambda
\end{array}\right)\right. \\
& -\frac{\pi t_{0}}{2 \lambda}\left\{\exp \left(\begin{array}{c}
-2 \pi x_{3} \\
\lambda
\end{array}\right)\right. \\
& \left.\left.\left.+\frac{\exp \left(2 \pi x_{3}^{+} / \lambda\right)+\cosh \left(2 \pi t_{0} / \lambda\right) \exp \left(2 \pi x_{3} / \lambda\right)}{\sinh \left(2 \pi t_{0} / h\right)}\right\}\right]\right\} \text {, }
\end{aligned}
$$

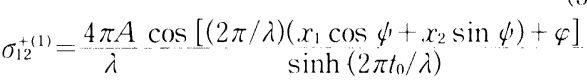

$$
\begin{aligned}
& \left\{\left[\frac{1-\nu}{2} \sigma_{1}^{\prime} \sin 2 \psi^{\prime}+\frac{1}{2} \tau^{\prime} \cos 2 \psi^{\prime}\right] \cosh \frac{2 \pi x_{3}^{-}}{\lambda}\right. \\
& -\frac{1}{2} \sigma_{1}^{\prime} \sin 2 \psi^{\prime}\left[\frac{\pi x_{3}^{-}}{\lambda} \sinh \left(\begin{array}{c}
2 \pi x_{3} \\
\lambda
\end{array}\right)\right. \\
& -\frac{\pi t_{0}}{2 \lambda}\left\{\exp \left(\frac{-2 \pi x_{3}}{\lambda}\right)\right. \\
& \left.\left.\left.+\frac{\exp \left(2 \pi x_{3}^{+} / \lambda\right)+\cosh \left(2 \pi t_{0} / \lambda\right) \exp \left(2 \pi x_{3} / \lambda\right)}{\sinh \left(2 \pi t_{0} / \lambda\right)}\right\}\right]\right\},
\end{aligned}
$$

where $x_{3}^{ \pm}$are the coordinates in thicksess direction measured from the top and bottom reference sufaces. respectively, and $t_{0}$ is the thickness between these two reference surfaces, which are defined by

$$
\begin{aligned}
& x_{3}^{+} \equiv \pm x_{3}-h_{0}^{ \pm}, \\
& t_{0} \equiv h_{0}^{+}-h_{0}^{-} .
\end{aligned}
$$

\section{2 Construction of three-dimensional solution}

In the previous subsection we obtained a two-dimensional first order solution for the undulating top surface. In the following, the solution with unit wave amplitude $(\mathrm{A}=1)$ will be denoted by $\sigma_{i j}^{+(1)}\left(\lambda, \psi^{\prime}, \varphi\left(\lambda, \psi^{\prime}\right)\right)$. Similarly, the two-dimensional fundamental solution for a sinusoidal bottom surface can be obtained, and denoted 
by $\sigma_{i j}^{-(1)}(\lambda, \psi, \varphi(\lambda, \psi))$.

Since the general form of an arbitrarily three-dimensional surface are expressed by Eqn. (3), the corresponding first order solutions will have the following form :

$$
\begin{aligned}
& \sigma_{i j}^{ \pm(1)}\left(x_{1}, x_{2}, x_{3}\right) \\
& =\int_{0}^{\infty} \int_{0}^{\pi} A^{ \pm}(\lambda, \psi) \sigma_{i j}^{ \pm(1)}(\lambda, \psi, \varphi(\lambda, \psi)) d \psi d \lambda
\end{aligned}
$$

We may discretize the integral representation of Eqn.

(3) in the following form;

$$
\begin{aligned}
& \zeta_{0}^{ \pm}\left(x_{1}, x_{2}\right)=\sum_{l=1}^{n} \sum_{k=1}^{m} A_{k l}^{ \pm} \cos \\
& {\left[\frac{2 \pi}{\lambda_{k}^{ \pm}}\left(x_{1} \cos \psi_{l}^{ \pm}+x_{2} \sin \psi_{l}^{+}\right)+\varphi_{k l}^{ \pm}\right]}
\end{aligned}
$$

where the wave lengths and wave directions are separated into $m$ and $n$ discrete components, respectively. Then, the solution corresponding to Eqn. (34) will be expressed by

$$
\sigma_{i j}^{ \pm(1)}\left(x_{1}, x_{2}, x_{3}\right)=\sum_{l=1}^{n} \sum_{k=1}^{m} A_{k l}^{+} \sigma_{i j}^{+(1)}\left(\lambda_{k}, \psi_{l}, \varphi_{k l}^{+}\right)
$$

It has been shown that the first order perturbation solution of a plate with arbitrarily undulated surfaces are obtained by the simple integration of Eqn. (33) which is based on the two dimensional solutions with out using a fundamental solution of a three-dimensional surface. This result stems entirely from the linearity of the first order solution with respect to the undulation. The validity of the proposed method will be discussed in the subsequent section.

\section{Illustrative Examples and Discussions}

In order to investigate the validity of the proposed method, comparisons are made with finite element results for two-dimensional problems. Then, relatively simple three-dimensional solutions are illustrated and compared with the analytical solution for a half-space based on a fully three-dimensional formulation.

We first consider a simple two-dimensional example, where the top surface of the plate is defined by

$$
\zeta_{0}^{+}\left(x_{1}, x_{2}\right)=A \cos \left[\begin{array}{c}
2 \pi \\
\lambda
\end{array}\left(x_{1} \cos \psi+x_{2} \sin \psi\right)\right] .
$$

In the following calculation we assume the symmetric surface undulation with respect to the middle surface.

Numerical results of the stress concentration factors at the wave trough are made in Table 1 for various wavelength/thickness and wave amplitude/wave-length

Table 1 Comparisons of numerical results

\begin{tabular}{clcccc}
\hline \multirow{2}{*}{$\lambda / \mathrm{t}_{6}$} & methods & \multicolumn{4}{c}{$\mathrm{A} / \lambda$} \\
& & 0.05 & 0.10 & 0.15 & 0.20 \\
\hline \multirow{2}{*}{2.0} & Perturbation & 1.592 & 2.184 & 2.777 & 3.369 \\
& FEM & 1.585 & 2.238 & 2.932 & 3.667 \\
\multirow{2}{*}{1.0} & Perturbation & 1.623 & 2.246 & 2.870 & 3.493 \\
& FEM & 1.624 & 2.221 & 2.787 & 3.351 \\
& Perturbation & 1.628 & 2.256 & 2.884 & 3.512 \\
\multirow{2}{*}{0.57} & FEM & 1.619 & 2.172 & 2.670 & 3.148 \\
& Perturbation & 1.628 & 2.257 & 2.885 & 3.513 \\
& FEM & 1.610 & 2.138 & 2.595 & 3.022 \\
& Perturbation & & & 2.885 & 3.513 \\
& FEM & & & 2.543 & 2.983 \\
\hline
\end{tabular}

ratios under uniaxial tension in the wave- direction, $i$. e. : $\sigma_{1}=1, \sigma_{2}=\tau=0$, and $\phi=0$. Comparing with the finite -element calculations by the use of ANSYS, it is confirmed that the proposed method is within few percent error for the range, $A / \lambda<0.2, \lambda / t_{0}>0.5$, and $A / t_{0}$ $<0.5$.

Next we consider the variation of stresses for various wave-directions, where the surface undulation parameters are selected as $A / \lambda=0.05$, and $\lambda / t_{0}=1.0$. The results are illustrated for the uniaxial loading condition $\left(\sigma_{1}=1, \sigma_{2}=\tau=0\right)$, and also for the pure shear loading condition $\left(\sigma_{1}=\sigma_{2}=0, \tau=1\right)$, in Figs. 4 and 5 , respectively. The results can be expressed by the combination of inplane and anti-plane two-dimensional solutions. In Fig. 4 we can also observe small tension in $x_{2}$-direction, which is caused by the effect of Poisson's ratio $(\nu=0.3)$.

Finaly we shall consider a simple but fully three-

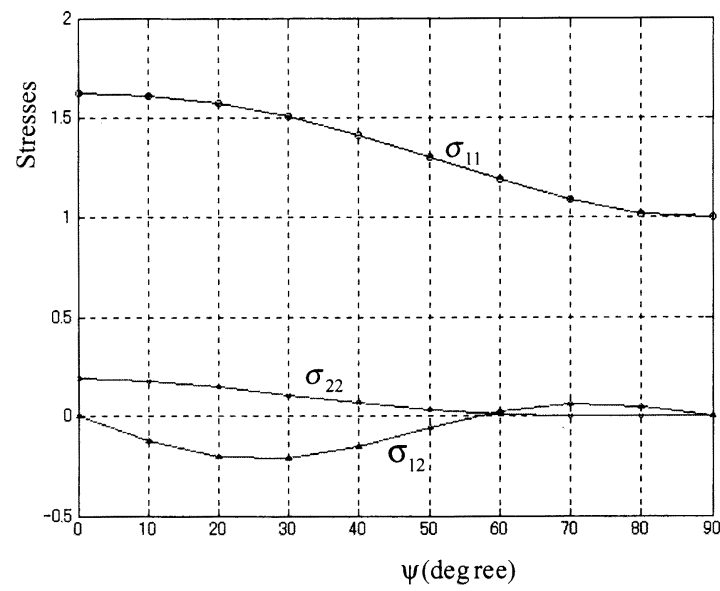

Fig. 4 Stresses $\sigma_{i j}$ for various $\psi$ at the trough of the surface under oblique uniaxial tention $\sigma_{1}=1, \sigma_{2}$ $=\tau=0$.

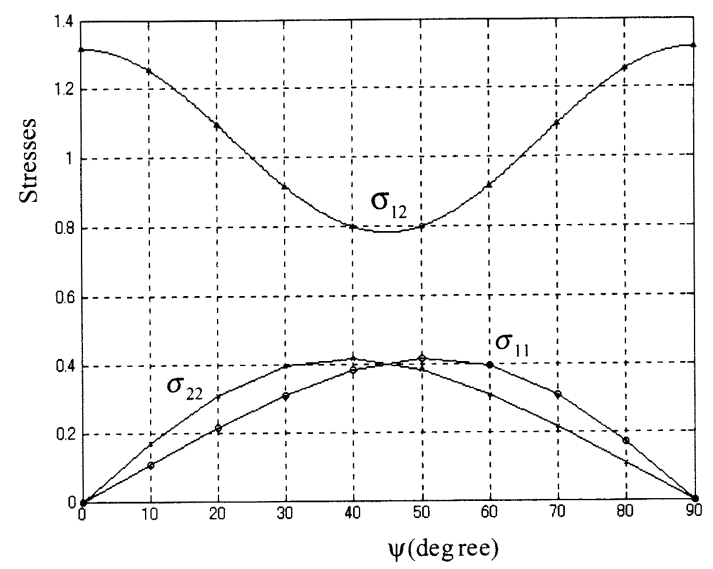

Fig. 5 Stresses $\sigma_{i j}$ for various $\psi$ at the trough of the surface under oblique pure shear $\sigma_{1}=\sigma_{2}=0, \tau=1$. 


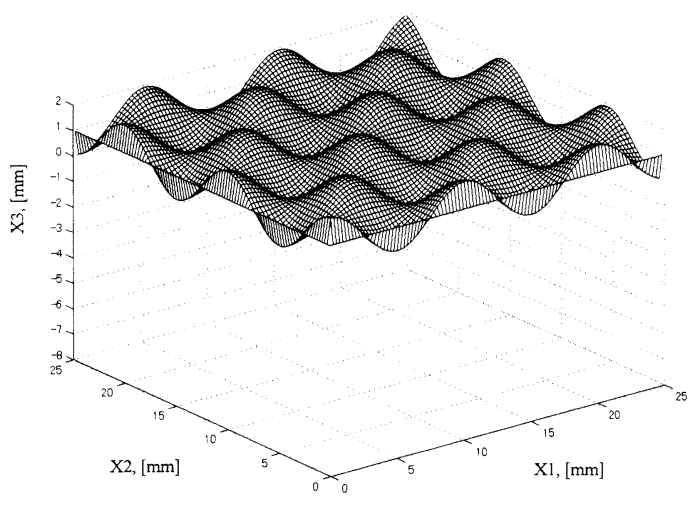

Fig. 6 Undulated three-dimensional surface, where $t_{0}$ $=10 \mathrm{~mm}$.

dimensional example, where the top surface of the plate is defined by

$$
\zeta_{0}^{+}\left(x_{1}, x_{2}\right)=A \cos \left(\frac{2 \pi x_{1}}{\lambda_{1}}\right) \cos \left(\frac{2 \pi x_{2}}{\lambda_{2}}\right),
$$

In this case, we also assume the symmetric undulation of the back surface with respect to the middle plane. The surface undulation parameters are selected as $A / \lambda_{1}$ $=A / \lambda_{2}=0.1$, and $\lambda_{1} / t_{0}=1.0$ (see Fig. 6). Obviously, Eqn. (37) can be decomposed into the two sinusoidal waves having two orthogonal wave-directions, $\psi=\pi / 4$ and $\psi=3 / 4 \pi$, so that the present method can construct a three-dimensional solution by the superposition of the two-dimensional solutions corresponding to these two waves. The uniform biaxial loading condition $\sigma_{1}=\sigma_{2}=$ 1 , and $\tau=0$ is applied, and the stress concentration factor for $\sigma_{11}$ and $\sigma_{22}$ at the trough point is obtained as 1.89 for $\nu=0$, and 2.15 for $\nu=0.3$, respectively. Gao ${ }^{11}$ obtained an analytical expression for a half-space with the same surface undulation based on the fully threedimensional formulation, which gives 1.89 for $\nu=0$, and 2.15 for $\nu=0.3$, respectively. Since the plate is relatively thick in the present example, the both results show extremely good agreement with each other.

\section{Conclusions}

A new solution method has been proposed for the analysis of an undulated plate of finite thickness subjected to in-plane loading conditions. The method treats the boundary conditions on the undulated surfaces by using a first order perturbation method, where the boundary conditions on the top and bottom surfaces are satisfied by the alternating solution procedure. Since an arbitrarily undulated surface of a plate may be approximated by the superpostion of two-dimensional sinusoidal waves having arbitrary wave lengths, wave directions and phases, it has been shown that only certain fundamental two-dimensional solutions are necessary to construct a fully three-dimensional solution. It should also be noted that the fundamental first order solution has been obtained in a closed form. The solution accuracy of the proposed method is examined by comparing the present results with finite-element solutions, and it has been confirmed that the proposed method is within few percent error for the range, $A / \lambda<$ $0.2, \lambda / t_{0}>0.5$, and $A / t_{0}<0.5$. Illustrative examples are also given for the stress analysis of a plate with slightly wavy surfaces in three-dimension, where the validity of the present formulation is confirmed by comparing with the results by the fully three-dimensional formulation.

One of the practical applications of the present method is the stress analysis of randomly undulated plates in marine structures, whose undulated surfaces are caused by corrosion. The surface characteristics of corroded plates of marine structures and the related stress analyses will appear in our subsequent paper.

\section{Acknowledgment}

During this work the first author has been in part supported by Grant-in-Aid for Scientific Research (Development Research B-10555338) from the Ministry of Education, Science and Culture to Yokohama National University. The second author has been awarded a scholarship for foreign students from the Ministry of Education, Science and Culture of Japan.

\section{References}

1) Gao, H., Stress concentration at slightly undulating surfaces, J. Mech. Phys. Solids, 39-4, 1991, pp. 443-458.

2) Sumi, Y., A second-order perturbation method for the stress analysis of solids with slightly wavy or irregular surfaces, JSME Int. J. -A, 38-4, 1995 , pp. 433-439.

3) Kantorovich, L. V. and Krylov, V. I., Approximate methods of higher analysis, Willey, New York, 1964, pp. 616-670. 